\title{
Circle maps with gaps: Understanding the dynamics of the two-process model for sleep-wake regulation $\dagger$
}

\author{
M. P. BAILEY, G. DERKS and A. C. SKELDON \\ Department of Mathematics, University of Surrey, Surrey GU2 7XH, UK \\ emails:matthew.bailey@surrey.ac.uk,g.derks@surrey.ac.uk, a.skeldon@surrey.ac.uk \\ (Received 25 September 2017; revised 20 March 2018; accepted 21 March 2018; first published online \\ 2 May 2018)

\begin{abstract}
For more than 30 years the 'two-process model' has played a central role in the understanding of sleep/wake regulation. This ostensibly simple model is an interesting example of a nonsmooth dynamical system, whose rich dynamical structure has been relatively unexplored. The two-process model can be framed as a one-dimensional map of the circle, which, for some parameter regimes, has gaps. We show how border collision bifurcations that arise naturally in maps with gaps extend and supplement the Arnold tongue saddle-node bifurcation set that is a feature of continuous circle maps. The novel picture that results shows how the periodic solutions that are created by saddle-node bifurcations in continuous maps transition to periodic solutions created by period-adding bifurcations as seen in maps with gaps.
\end{abstract}

Key words: 37E10 Maps of the circle; 92B25 Biological rhythms and synchronisation; 37G15 Bifurcations of limit cycles and periodic orbits; 37E05 Maps of the interval; 37N25 Dynamical systems in biology.

\section{Introduction}

Since the seminal paper of Arnold (1991), circle maps have played an important role in understanding the behaviour of a variety of physical and biological systems. The circle maps that Arnold considered were monotonic and could be discontinuous. Nondecreasing circle maps with gaps were also studied by Keener (1980) and Rhodes \& Thompson (1986), who proved that solutions could only be either periodic or aperiodic. Furthermore, that if the map depended smoothly and monotonically on a parameter, then the periodic solutions occur in intervals with a devil's staircase structure dependence on the parameter.

As described in the extensive review paper by Granados et al. (2017), the same sequences of periodic solutions have been repeatedly observed in monotonic maps with gaps of the real line, and have been termed period-adding sequences in the non-smooth literature,

$\dagger$ This work was partially supported by the Engineering and Physical Sciences Research Council (Grant number EP/M506655/1). 
for example, see Avrutin et al. (2006). The observation of period-adding sequences is typically linked with border collision bifurcations where fixed points of the map are created/destroyed by colliding with the gap.

In this paper, we describe an interesting application that can be framed as a map of the circle. As a parameter is varied, this map transitions from a continuous monotonic circle map to a monotonic circle map with gaps. This map enables us to understand how border collisions in gap maps can play a similar role to saddle-node bifurcations in continuous circle maps in forming boundaries for existence regions of periodic solutions. This view provides an interesting link between the Arnold tongue picture for continuous maps where resonant regions are bounded by saddle-node bifurcations and the sequences of period-adding bifurcations commonly seen in the maps with gaps literature.

The application arises in the context of sleep/wake regulation. Understanding sleep/wake regulation is important because chronic sleep restriction or mis-timed sleep have been shown to disrupt the carefully orchestrated approximately daily (circadian) rhythms that govern many physiological processes, see Möller-Levet et al. (2013) and Archer et al. (2014). Furthermore, poor sleep has been correlated with a wide range of health issues including depression, psychotic disorders, neurodegenerative conditions, disease progression in cancer, cardiovascular disease, obesity and diabetes, for example, see Knutson (2010), Nielsen et al. (2011) and Luyster et al. (2012). Mathematical modelling has been used as a tool to help understand biological mechanisms of sleep for decades, with the two-process model of particular importance. The two-process model was first described in Borbély (1982) and extended in Daan et al. (1984) and has since provided a theoretical framework and even the language now commonly used to describe sleep-wake regulation: the original paper by Borbély (1982) has been cited more than 3,000 times.

With greater understanding of the neuronal mechanisms that underlie sleep/wake regulation, as described by Saper et al. (2005), has come more sophisticated mathematical models, such as those of Tamakawa et al. (2006), Phillips \& Robinson (2007), Diniz Behn et al. (2007), Postnova et al. (2009), Diniz Behn \& Booth (2010), Rempe et al. (2010) and Kumar et al. (2012). A discussion of those models can be found in the review by Booth \& Diniz Behn (2014). However, the two-process model remains at the heart of many of these models: in Skeldon et al. (2014), it is shown that the model introduced in Phillips \& Robinson (2007) can be formally reduced to the two-process model using multiple timescale analysis and the relation of the two-process model to more general neuronal models is discussed in Skeldon et al. (2017b).

Consequently understanding the dynamics of the two-process model is important for two reasons. First, because of the central role of this model in the understanding of sleep/wake regulation, and second, in providing a deeper understanding of some of the interesting dynamical phenomena that have been observed in neuronal models of sleep/wake regulation.

The two-process model proposes that the sleep-wake cycle can be understood in terms of the interaction of two oscillatory processes: a homeostatic sleep process and a circadian process. The homeostatic sleep process results in a homeostatic sleep pressure that can be viewed as the physiological need for sleep. The sleep pressure increases monotonically during wake and decreases during sleep. Switching between the sleep and wake states occurs at threshold values of the sleep pressure, with the transition from wake to sleep 

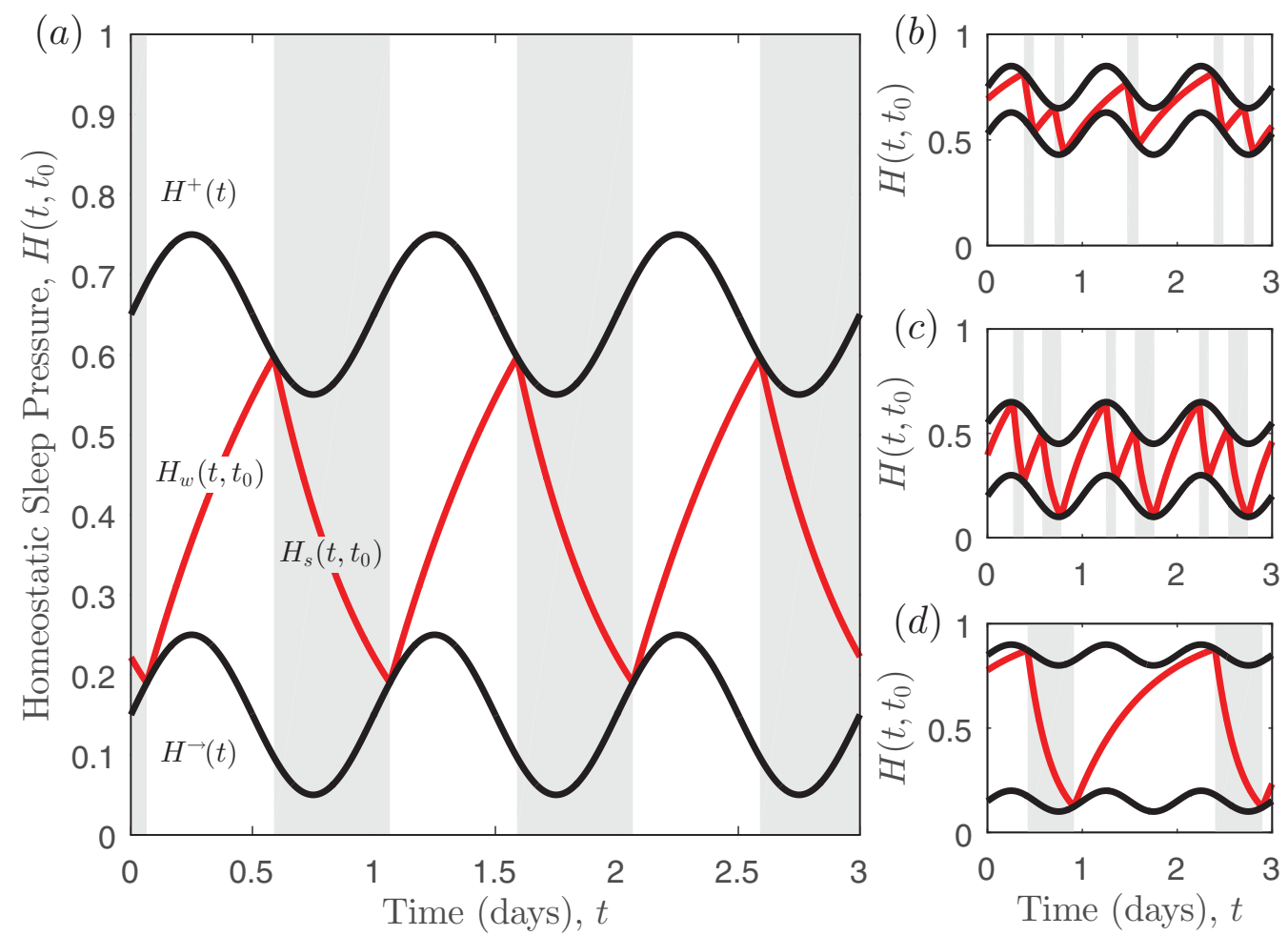

FIgURE 1. The two-process model. (a) A one sleep/day periodic sleep-wake cycle, showing the homeostatic sleep pressure (shown in red) increasing during wake until it hits the upper threshold and then decreasing during sleep (shaded regions) until it hits the lower threshold. The thresholds are shown in black. Parameter values: $a=0.1, H_{0}^{-}=0.15, H_{0}^{+}=0.65, \chi_{s}=0.417, \chi_{w}=0.75$. Panels (b)-(d) show three other possible patterns that occur as parameters are varied. Parameter values: (b) $a=0.1, H_{0}^{-}=0.53, H_{0}^{+}=0.75, \chi_{s}=0.25, \chi_{w}=0.75$ giving a sleep-wake cycle that repeats every two days with three sleep episodes; (c) $a=0.1, H_{0}^{-}=0.2, H_{0}^{+}=0.55, \chi_{s}=0.124, \chi_{w}=0.5$ giving a sleep-wake cycle that repeats every day with two sleep episodes; (d) $a=0.05, H_{0}^{-}=0.15, H_{0}^{+}=$ $0.85, \chi_{s}=0.25, \chi_{w}=0.75$ giving a sleep-wake cycle that repeats every two days with one sleep episode.

occurring at the upper threshold and from sleep to wake at the lower threshold. Both thresholds are modulated by the circadian oscillation, as illustrated in Figure 1.

As the examples shown in Figure 1 illustrate and noted by Daan et al. (1984), this deceptively simple model can display a wide range of different sleep-wake patterns. Here, we seek to shed light on the bifurcation structures associated with the transitions between different kinds of pattern. It was shown by Nakao et al. (1997) and Nakao \& Yamamoto (1998) and subsequently discussed further by Skeldon et al. (2014) that the two-process model can be represented as a one-dimensional map with gaps. The inherent periodicity of the circadian process means that this one-dimensional map can be linked to a circle map. Consequently, both classic results for circle maps and well-known results for maps with gaps will be relevant to our discussion.

We note that although simulations suggest that the two-process model exhibits a rich variety of sleep/wake patterns and its representation as a one-dimensional map has 
also been identified before, there has been no detailed mathematical analysis of the model. Given its prominence and importance for the field of sleep/wake regulation, this is surprising. We would argue that understanding the mathematical structure is a critical step to fully understand its strengths and weaknesses as a model of observed sleep behaviour. Interestingly, our analysis also brings insight into the bifurcation structures that occur in circle maps as they transition from being continuous to discontinuous.

The layout of the paper is as follows. In Section 2, we formally define the twoprocess model and its associated one-dimensional map and give some basic properties. In Section 3, we show that there is a parameter regime where the one-dimensional map is a lift of a continuous monotonic circle map and hence the regions of existence of different types of periodic solutions form Arnold tongues bounded by saddle-node bifurcations. In Section 4, we find the parameter regions for which gaps in the map occur and monotonicity is preserved, and describe the structure of the gap. In Section 5, we show that discontinuities in the map result in border collision bifurcations. Both saddle-node bifurcations and border collision bifurcations are mechanisms for the creation/destruction of periodic solutions. We show how the border collisions and period-adding bifurcations relate to the tongue structure formed by the saddle-node bifurcations, hence, forming a novel picture of how the intervals of periodic solutions in continuous circle maps relate to those in maps with gaps. We end with a discussion section that summarises our key results and discusses the biological relevance.

\section{Definition of the model and $(p, q)$ periodic orbits}

\subsection{Definition of the two-process model and associated map}

As described in Section 1 and illustrated in Figure 1, the two-process model consists of a homeostatic sleep pressure that increases during wake and decreases during sleep with switching between wake/sleep and sleep/wake occurring at threshold values.

We define $H_{s}\left(t, t_{0}\right)$ to be the homeostatic sleep pressure during sleep, which starts at $t=t_{0}$ on the upper threshold $H^{+}(t)$, and $H_{w}\left(t, t_{0}\right)$ to be the homeostatic sleep pressure during wake, which starts at $t=t_{0}$ on the lower threshold $H^{-}(t)$. Then, the expressions for $H_{s}$ and $H_{w}$ are

$$
H_{s}\left(t, t_{0}\right):=H^{+}\left(t_{0}\right) e^{\frac{\left(t_{0}-t\right)}{\chi_{s}}} \text { and } H_{w}\left(t, t_{0}\right):=1-\left(1-H^{-}\left(t_{0}\right)\right) e^{\frac{\left(t_{0}-t\right)}{\chi_{w}}} .
$$

Here, the upper and lower thresholds are given by

$$
H^{+}(t)=H_{0}^{+}+a \sin (2 \pi t) \text { and } H^{-}(t)=H_{0}^{-}+a \sin (2 \pi t) .
$$

Defined in this way the model has five parameters: sleep and wake time constants $\chi_{s}, \chi_{w}$, which determine the rate of dissipation and build-up of homeostatic sleep pressure; the mean values of the upper and lower thresholds, $\mathrm{H}_{0}^{+}$and $\mathrm{H}_{0}^{-}$, respectively, and the circadian amplitude, $a$. The period of the circadian oscillator has been scaled to one and the sleep and wake homeostatic pressure are scaled such that they would asymptote to 0 and 1, respectively, in the absence of the thresholds. Biological relevance places some restrictions on physiologically plausible values. Specifically, requiring $H_{s}$ to monotonically 
decrease, $H_{w}$ to monotonically increase and the homeostatic sleep pressure to start and stay in the interval $(0,1)$ for any initial value on the upper or lower threshold leads to $\chi_{s}>0, \chi_{w}>0$,

$$
a<H_{0}^{-}<H_{0}^{+}<1-a,
$$

where without loss of generality we consider $a \geqslant 0$.

This paper focuses on understanding the regions of existence of periodic orbits in the two-process model and the associated bifurcations. In order to study periodic orbits, it is sufficient to consider the map that takes a point $\left(H_{0}, t_{0}\right)$ on the upper threshold and maps it into the next point on the upper threshold. Since the upper threshold satisfies $H_{0}=H^{+}\left(t_{0}\right)$, this is in fact a one-dimensional map. An equivalent description (as far as periodic orbits is concerned) is given by the map that takes a point on the lower threshold to the next point on the lower threshold.

Although it is perhaps easiest to describe the map in terms of the homeostatic sleep pressure, as above, to understand the dynamics, we define the map in terms of time. The map from the upper threshold into itself can be regarded as a composition of two maps. One map goes 'down' from the upper to the lower threshold and the other map goes 'up' from the lower to the upper threshold. This motivates the definition of the down map $T_{d}: \mathbb{R} \rightarrow \mathbb{R}$ and the up map $T_{u}: \mathbb{R} \rightarrow \mathbb{R}$, where $T_{d}\left(t_{0}\right)$ and $T_{u}\left(t_{0}\right)$ are the first times greater than $t_{0}$ such that

$$
H_{s}\left(T_{d}\left(t_{0}\right), t_{0}\right)=H^{-}\left(T_{d}\left(t_{0}\right)\right) \quad \text { and } \quad H_{w}\left(T_{u}\left(t_{0}\right), t_{0}\right)=H^{+}\left(T_{u}\left(t_{0}\right)\right) \text {. }
$$

The composite map that maps the upper threshold into itself is $T_{s}: \mathbb{R} \rightarrow \mathbb{R}$ with

$$
T_{s}\left(t_{0}\right)=T_{u}\left(T_{d}\left(t_{0}\right)\right)
$$

The periodicity of the upper and lower threshold, (2.2) imply that all three maps satisfy

$$
T_{i}\left(t_{0}+1\right)=T_{i}\left(t_{0}\right)+1, \quad i=d, u, s .
$$

Typical examples of the three maps, $T_{i}, i=d, u, s$ are shown in Figure 2.

For large regions of the parameter plane, including small values of the parameter $a$, the map $T_{S}$ is monotonic. In these regions, the relation (2.6) implies that $T_{S}$ can be seen as the degree 1 lift of a circle map on the interval [0,1], and hence the theory of monotonic circle maps with and without gaps can be applied. Thus, the map has a unique rotation number given by

$$
\tilde{\rho}\left(T_{s}\right)=\lim _{n \rightarrow \infty} \frac{T_{s}^{n}\left(t_{0}\right)-t_{0}}{n}
$$

This rotation number does not depend on the value $t_{0}$. If the rotation number is rational, then the map has periodic solutions, see Rhodes \& Thompson (1986) for more details.

\subsection{Definition of a $(p, q)$ periodic solution}

The example trajectories shown in Figure 1 suggest that there are many different types of periodic orbits. These can be characterised as having $p$ sleeps (and hence $p$ wakes) in $q$ 

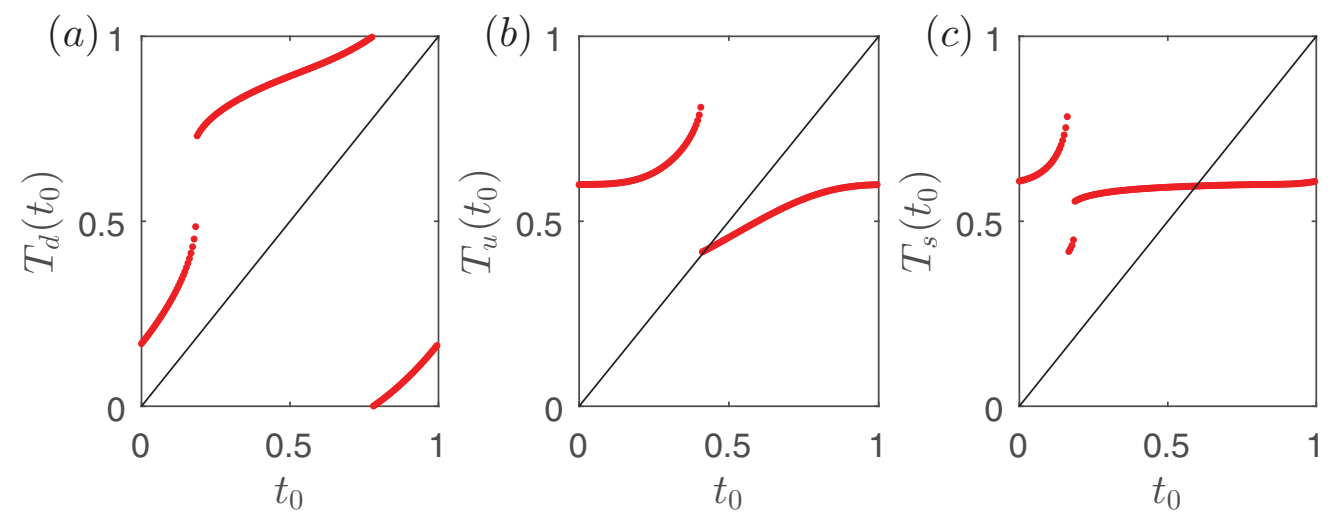

Figure 2. Typical examples of $T_{i} \bmod 1$ for $i=d$, $u$, s. Parameter values: $a=0.15, H_{0}^{-}=0.25, H_{0}^{+}=$ $0.75, \chi_{s}=0.25, \chi_{w}=0.75$. For the remainder of the examples in this paper, we take $H_{0}^{+}, \chi_{s}, \chi_{s}$ to be the values given here, but $a$ and $H_{0}^{-}$will vary.

days. Hence, we say that $t_{0}$ generates a $(p, q)$ periodic orbit if

$$
T_{s}^{p}\left(t_{0}\right)=t_{0}+q ; \quad\left(T_{s}^{j}\left(t_{0}\right)-t_{0}\right) \notin \mathbb{N} \quad \text { for } \quad j=1, \ldots, p-1 .
$$

For our parameter values, the map $T_{s}$ will be monotonic, and the periodicity relation (2.6) implies that the set $T_{s}([0,1])$ will be contained in an interval of length 1 . Thus, if $T_{s}$ has a $(p, q)$ periodic orbit, the greatest common divisor of $p$ and $q$ will be 1 and the theory of monotonic circle maps gives that function $T_{S}$ has the rotation number $\tilde{\rho}\left(T_{S}\right)=\frac{q}{p}$, see the papers by Arnold (1991), Keener (1980) and Rhodes \& Thompson (1986) for details.

Having defined the map and $(p, q)$ periodic solutions, we move on to analyse the bifurcations of these periodic solutions.

\section{Saddle-node bifurcations}

For circadian amplitude $a=0$, the biological constraints (2.3) become $0<H_{0}^{-}<H_{0}^{+}<1$. The dynamics of the homeostatic sleep pressure gives that any $t_{0} \in[0,1]$ results in a solution that repeats with a 'natural' period $T_{\text {nat }}$ given by

$$
T_{\text {nat }}=\chi_{s} \log \left(\frac{H_{0}^{+}}{H_{0}^{-}}\right)+\chi_{w} \log \left(\frac{1-H_{0}^{-}}{1-H_{0}^{+}}\right),
$$

where $\chi_{s} \log \left(\frac{H_{0}^{+}}{H_{0}^{-}}\right)$is the length of each sleep episode and $\chi_{w} \log \left(\frac{1-H_{0}^{-}}{1-H_{0}^{+}}\right)$is the length of each wake episode. Such solutions are $(p, q)$-periodic solutions when

$$
T_{\text {nat }}=\frac{q}{p} \text { and } \operatorname{gcd}(p, q)=1 .
$$

The natural period $T_{\text {nat }}$ is monotonically decreasing in the parameter $H_{0}^{-}$and monotonically increasing in the parameters $H_{0}^{+}, \chi_{s}$ and $\chi_{w}$. Thus, for $a=0$, the map $T_{s}$ can be written as $T_{s}\left(t_{0}\right)=t_{0}+T_{\text {nat }}$ and generates a twist map in $t_{0}$ and any one of these four 

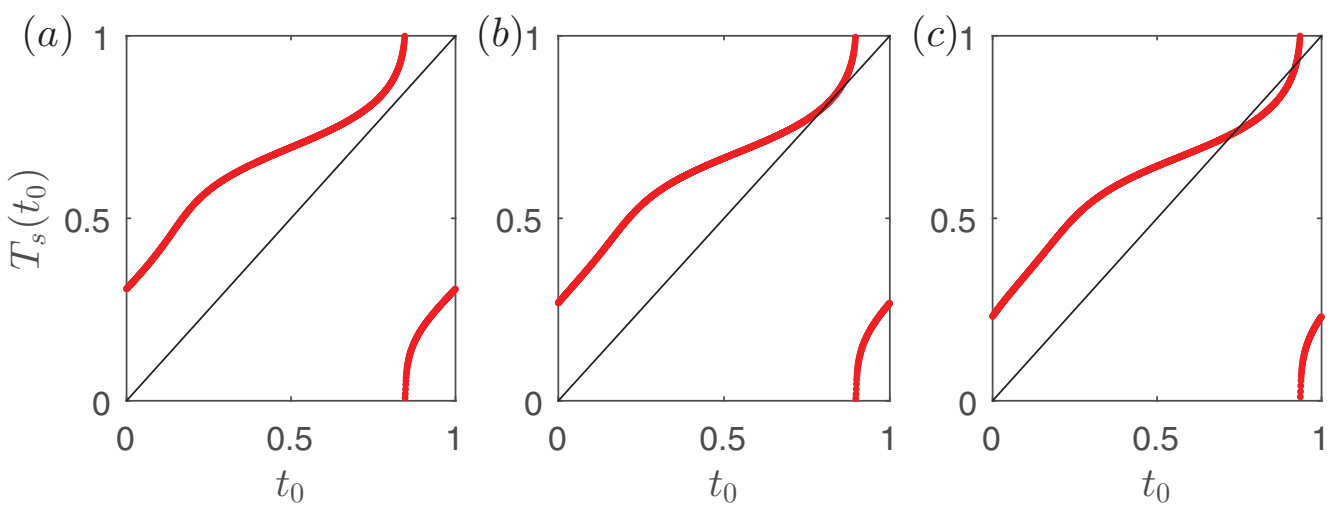

FIGURE 3. The creation of a pair of $(p, q)=(1,1)$ solutions via a saddle-node bifurcation for $a=0.05$ and $H_{0}^{-}$varying. (a) No fixed points, $H_{0}^{-}=0.177$; (b) at the saddle-node bifurcation, $H_{0}^{-}=0.197$; (c) two fixed points, $H_{0}^{-}=0.217$. Note that for these parameter values, the primary effect of $H_{0}^{-}$is to shift the position of the map rather than change its gradient.

parameters. The sleep map $T_{s}$ depends smoothly on $a$; hence, perturbation theory gives that also for small $a$, the map $T_{s}$ is monotonic in $t_{0}$ and is conjugate to the degree 1 lift of a monotonic, continuous Arnold circle map. Therefore, regions of $(p, q)$ periodic orbits of the map persist for small amplitude $a$, forming tongue-like regions (Arnold tongues, see Arnold, 1991) in a two-dimensional parameter plane consisting of one of the four parameters $\chi_{s}, \chi_{w}, H_{0}^{+}$or $H_{0}^{-}$along the horizontal axis and the circadian amplitude $a$ along the vertical axis. At the edge of each tongue are saddle-node bifurcations, which create a pair of solutions, one stable and one unstable. For illustration, a sequence of maps showing the transition from no fixed points to a pair of solutions is shown in Figure 3.

In the remainder of this paper, we consider the bifurcation behaviour as a function of $a$ and $H_{0}^{-}$. Since $T_{\text {nat }}$ is a monotonic function of $\chi_{s}, \chi_{w}$ and $H_{0}^{+}$, the results will be qualitatively similar if any of the latter three parameters were selected instead of $H_{0}^{-}$.

In this section, we first consider a small $a$ analysis of the primary $(p, q)=(1, q)$ tongues before numerically finding general $(p, q)$-tongues.

\subsection{Small circadian amplitude approximation for $(p, q)=(1, q)$ tongue}

The analysis in this section focuses on the case where $p=1$. Writing the length of the sleep episode as $\tau:=T_{d}\left(t_{0}\right)-t_{0} \in(0, q)$, the homeostatic sleep pressure in a $(1, q)$ periodic trajectory can be described as follows: being in the sleep state from $t_{0}$ to $t_{0}+\tau$; hitting the lower threshold $\mathrm{H}^{-}$at $t_{0}+\tau$ and switching to the wake state; in the wake state from $t_{0}+\tau$ to $t_{0}+q$, with a further switch at $t_{0}+q$ when it reaches the upper threshold $\mathrm{H}^{+}$. This gives the relations

$$
H_{s}\left(t_{0}+\tau, t_{0}\right)=H^{-}\left(t_{0}+\tau\right) \quad \text { and } \quad H_{w}\left(t_{0}+q, t_{0}+\tau\right)=H^{+}\left(t_{0}+q\right)=H^{+}\left(t_{0}\right) .
$$


Using the definition of the sleep and wake forms of the homeostatic sleep pressure in (2.1), this can be rewritten as

$$
H^{+}\left(t_{0}\right)=\frac{1-e^{\frac{\tau-q}{\chi_{w}}}}{1-e^{-\frac{\tau}{\chi_{s}}} e^{\frac{\tau-q}{\chi_{w}}}} \quad \text { and } \quad H^{-}\left(t_{0}+\tau\right)=H^{+}\left(t_{0}\right) e^{-\frac{\tau}{\chi_{s}}}
$$

Introducing the functions

$$
\alpha(\tau ; q)=\frac{1-e^{\frac{\tau-q}{\chi_{w}}}}{1-e^{-\frac{\tau}{\chi_{s}}} e^{\frac{\tau-q}{\chi_{w}}}} \text { and } \beta\left(\tau, t_{0}\right)=H^{+}\left(t_{0}\right) e^{-\tau / \chi_{s}}-a \sin \left(2 \pi\left(t_{0}+\tau\right)\right),
$$

and using the definition of $H^{-}(t)$, this implies that if a $(1, q)$ periodic orbit exists, then there is a pair $\left(t_{0}, \tau\right) \in(0,1] \times(0, q)$ satisfying

$$
H^{+}\left(t_{0}\right)=\alpha(\tau ; q)
$$

and

$$
H_{0}^{-}=\beta\left(\tau, t_{0}\right)
$$

The function $\alpha(\tau ; q)$ should be considered as a function of $\tau$; the parameter $q$ is added to stress its dependence on this parameter. It is straightforward to show that $\alpha(\tau ; q)$ is a monotonically decreasing function for $\tau \in[0, q]$ with $\alpha(0)=1$ and $\alpha(q)=0$. In other words, $\alpha$ is a bijection between $[0, q]$ and $[0,1]$. The biological constraints $(2.3)$ imply that the range of the upper threshold $H^{+}$lies fully in the interval $[0,1]$. Using the monotonicity of $\alpha$, we can use (3.2) to define a function $\tau:[0,1] \rightarrow[0, q]$ with

$$
\alpha\left(\tau\left(t_{0}\right) ; q\right)=H^{+}\left(t_{0}\right)
$$

This definition, the relation $H^{+}\left(t_{0}\right)=H_{0}^{+}+a \sin \left(2 \pi t_{0}\right)$ and (3.3) show that for any

$$
H_{0}^{-} \in\left\{\beta\left(\tau\left(t_{0}\right), t_{0}\right) \mid t_{0} \in[0,1]\right\},
$$

there are two $t_{0} \in[0,1]$ such that the pair $\left(t_{0}, \tau\left(t_{0}\right)\right)$ satisfies equations (3.2) and (3.3). If $T_{s}$ is continuous (which it is for small $a$ ), then these two pairs $\left(t_{0}, \tau\left(t_{0}\right)\right)$ correspond to periodic solutions.

For $a=0, H^{+}\left(t_{0}\right)=H_{0}^{+}$for all $t_{0}$. Furthermore, $H_{0}^{+} \in(0,1)$ implies that there exists a unique $\tau_{0}$ such that

$$
\alpha\left(\tau_{0} ; q\right)=H_{0}^{+} \text {. }
$$

Thus, equation (3.2) gives that at $a=0, \tau\left(t_{0}\right)=\tau_{0}$, for all $t_{0} \in(0, q)$, and (3.3) implies that for

$$
H_{0}^{-}=H_{0}^{+} e^{-\frac{\tau_{0}}{\gamma_{s}}},
$$

any $t_{0} \in(0, q)$ generates a $(1, q)$ periodic solution. To see that this is equivalent to $T_{\text {nat }}=q$ with $T_{\text {nat }}$ given by (3.1), we first observe that the relation above between $H_{0}^{-}$and $H_{0}^{+}$ implies that the length of the sleep period satisfies $\tau_{0}=\chi_{s} \log \left(\frac{H_{0}^{+}}{H_{0}^{-}}\right)$. Next, we use the relations $\alpha\left(\tau_{0} ; q\right)=H_{0}^{+}$and $e^{-\frac{\tau_{0}}{\chi_{s}}}=H_{0}^{-} / H_{0}^{+}$to see that the length of the wake period satisfies $q-\tau_{0}=\chi_{w} \log \left(\frac{1-H_{0}^{-}}{1-H_{0}^{+}}\right)$, and therefore from (3.1), $T_{\text {nat }}=q$. 
Having established that $H_{0}^{-}=H_{0}^{+} e^{-\frac{\tau_{0}}{\gamma_{s}}}$ leads to a $(1, q)$ periodic solution at $a=0$, we will now use (3.5) to determine a small $a$ approximation for the interval of $H_{0}^{-}$values near $H_{0}^{+} e^{-\frac{\tau_{0}}{\chi_{s}}}$ for which $(1, q)$ periodic orbits exist. For $a$ small, the function $\tau\left(t_{0}\right)$ can be expanded as

$$
\tau\left(t_{0}\right)=\tau_{0}+a \tau_{1}\left(t_{0}\right)+\mathcal{O}\left(a^{2}\right) .
$$

Using the definition of $\tau$ (3.4), it follows immediately that

$$
\tau_{1}=\frac{\sin \left(2 \pi t_{0}\right)}{\alpha^{\prime}\left(\tau_{0} ; q\right)}
$$

Now, (3.3) gives the range of $H_{0}^{-}$values for which $(1, q)$ periodic orbit exist, parameterised by $t_{0}$ :

$$
H_{0}^{-}=\beta\left(\tau\left(t_{0}\right), t_{0}\right)=\left[H_{0}^{+}+a \sin \left(2 \pi t_{0}\right)\right] e^{-\frac{\tau\left(t_{0}\right)}{\chi_{s}}}-a \sin \left(2 \pi\left(t_{0}+\tau\left(t_{0}\right)\right)\right) .
$$

Using the expansion $\tau\left(t_{0}\right)=\tau_{0}+\frac{a \sin \left(2 \pi t_{0}\right)}{\alpha^{\prime}\left(\tau_{0} ; q\right)}+\mathcal{O}\left(a^{2}\right)$, we get

$$
H_{0}^{-}=H_{0}^{+} e^{-\frac{\tau_{0}}{\chi_{s}}}+a\left(e^{-\frac{\tau_{0}}{\chi_{s}}} \sin \left(2 \pi t_{0}\right)\left(1-\frac{H_{0}^{+}}{\alpha^{\prime}\left(\tau_{0} ; q\right)}\right)-\sin \left(2 \pi\left(\tau+t_{0}\right)\right)\right)+\mathcal{O}\left(a^{2}\right) .
$$

This can be written as

$$
H_{0}^{-}\left(a, t_{0}\right)=H_{0}^{+} e^{-\frac{\tau_{0}}{\chi_{s}}}+a \rho \sin \left(2 \pi\left(t_{0}+\theta\right)\right)+\mathcal{O}\left(a^{2}\right)
$$

where

$$
\rho \cos (\theta)=\left(e^{-\frac{\tau_{0}}{\chi_{s}}}\left(1-\frac{H_{0}^{+}}{\chi_{s} \alpha^{\prime}\left(\tau_{0} ; q\right)}\right)-\cos \left(2 \pi \tau_{0}\right)\right) \quad \text { and } \quad \rho \sin (\theta)=\sin \left(2 \pi \tau_{0}\right),
$$

implying

$$
\rho=\left[\sin ^{2}\left(2 \pi \tau_{0}\right)+\left(e^{-\frac{\tau_{0}}{\chi_{s}}}\left(1-\frac{H_{0}^{+}}{\chi_{s} \alpha^{\prime}\left(\tau_{0} ; q\right)}\right)-\cos \left(2 \pi \tau_{0}\right)\right)^{2}\right]^{\frac{1}{2}} .
$$

This shows that for $a$ small, two $(1, q)$ periodic orbits exist in a wedge with $H_{0}^{-} \in$ $\left[H_{0}^{+} e^{-\frac{\tau_{0}}{\chi_{s}}}-a \rho, H_{0}^{+} e^{-\frac{\tau_{0}}{\chi_{s}}}+a \rho\right]+\mathcal{O}\left(a^{2}\right)$, as illustrated in Figure 4(a). At the edges of the wedge, the periodic solutions disappear in a saddle-node bifurcation.

\subsection{Calculation of general $(p, q)$ tongues}

For $a$ small, the circle map nature of the map of the two-process model means that there exist regions of periodic solutions bounded by saddle-node bifurcations. In order to compute the boundary of the tongue for any $(p, q)$-periodic orbit, we therefore use the condition for periodicity along with the fact that at saddle-node bifurcations the map has gradient one. Hence, for fixed $\chi_{s}, \chi_{w}, H_{0}^{+}, a$, we seek the values of $\left(t_{0}, H_{0}^{-}\right)$such that

$$
T_{s}^{p}\left(t_{0}\right)=t_{0}+q
$$



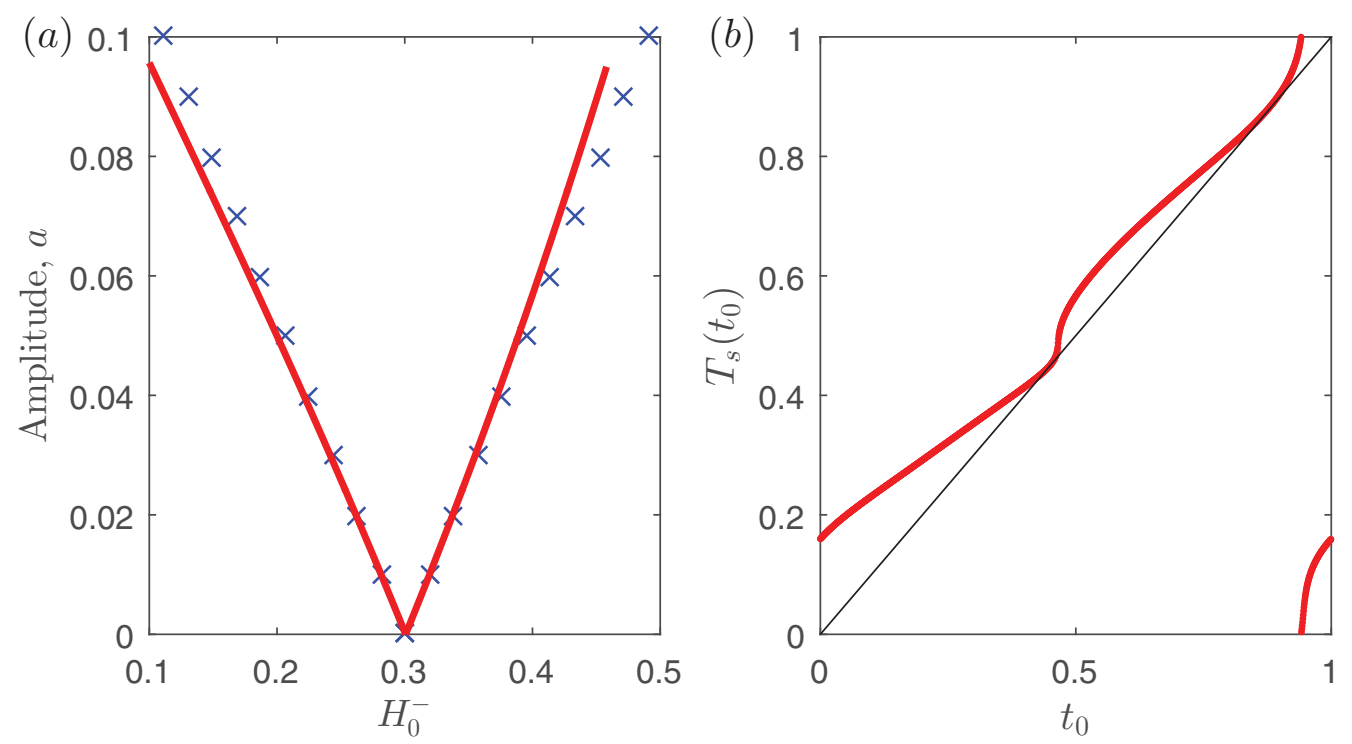

Figure 4. (a) A comparison of the numerical computation of the Arnold tongues for $(p, q)=(1,1)$ (solid lines) and the analytical expression for small $a$ given in equation (3.7) (crosses). (b) The map of the two-process model for $a=0.05$ and $H_{0}^{-}=0.55$, where a saddle-node bifurcation for the $(2,1)$ periodic solutions occurs.

and

$$
\left(T_{s}^{p}\right)^{\prime}\left(t_{0}\right)=\prod_{j=0}^{p-1} T_{u}^{\prime}\left(T_{d}\left(T_{s}^{j}\left(t_{0}\right)\right)\right) T_{d}^{\prime}\left(T_{s}^{j}\left(t_{0}\right)\right)=1,
$$

where we use the notation $T_{s}^{0}\left(t_{0}\right)=t_{0}$. This enables all $(p, q)$ tongues, with multiple sleeps across any number of days, to be found. In Figure 4(a), a comparison of the numerical computation of the $(1,1)$ tongue with the analytical expression given in equation $(3.7)$ is shown. As expected, both the numerical solution and analytical expression agree for small $a$, but diverge as $a$ becomes larger. Also note that for a $(p, q)$-periodic solution there will be $p$ values of $t_{0} \in(0,1]$ that lead to the same solution, as illustrated for $(p, q)=(2,1)$ in Figure 4(b).

In Figure 5, the saddle-node bifurcation curves are plotted for a range of $(p, q)$ periodic orbits. The (red) dashed lines mark the boundaries of the biological constraints on the parameters, see (2.3). The solid (red) lines mark the saddle-node bifurcations. Note that the right-hand boundary of each tongue comes to an end, at this point, the saddle-node bifurcations cease to exist. The explanation for this will follow in Section 5. As is known for circle maps, a tongue exists for each pair of values $(p, q), \operatorname{gcd}(p, q)=1$, although only a few of the largest tongues are plotted in Figure 5(a).

To further illustrate the sequence of bifurcations for increasing $H_{0}^{-}$and the fact that the parameter dependence of the rotation numbers has a devil's staircase structure, a bifurcation diagram for a slice through the bifurcation set for $a=0.05$ is shown in Figure 5(b). For small circadian amplitudes (such as $a=0.05$ as used in Figure 5), the map is continuous and periodic solutions are created/destroyed by saddle-node bifurcations. 


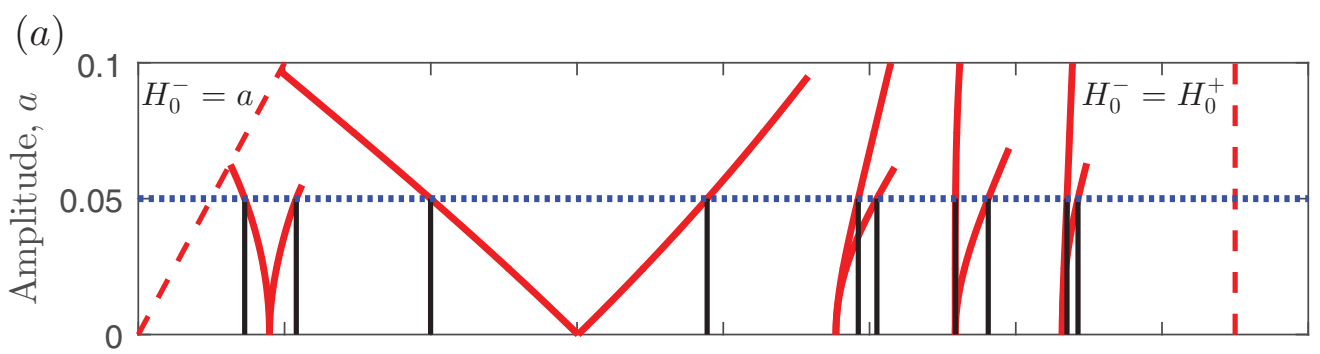

$(2,3)$

$(1,1)$

$(3,2)$

$(2,1) \quad(3,1)$

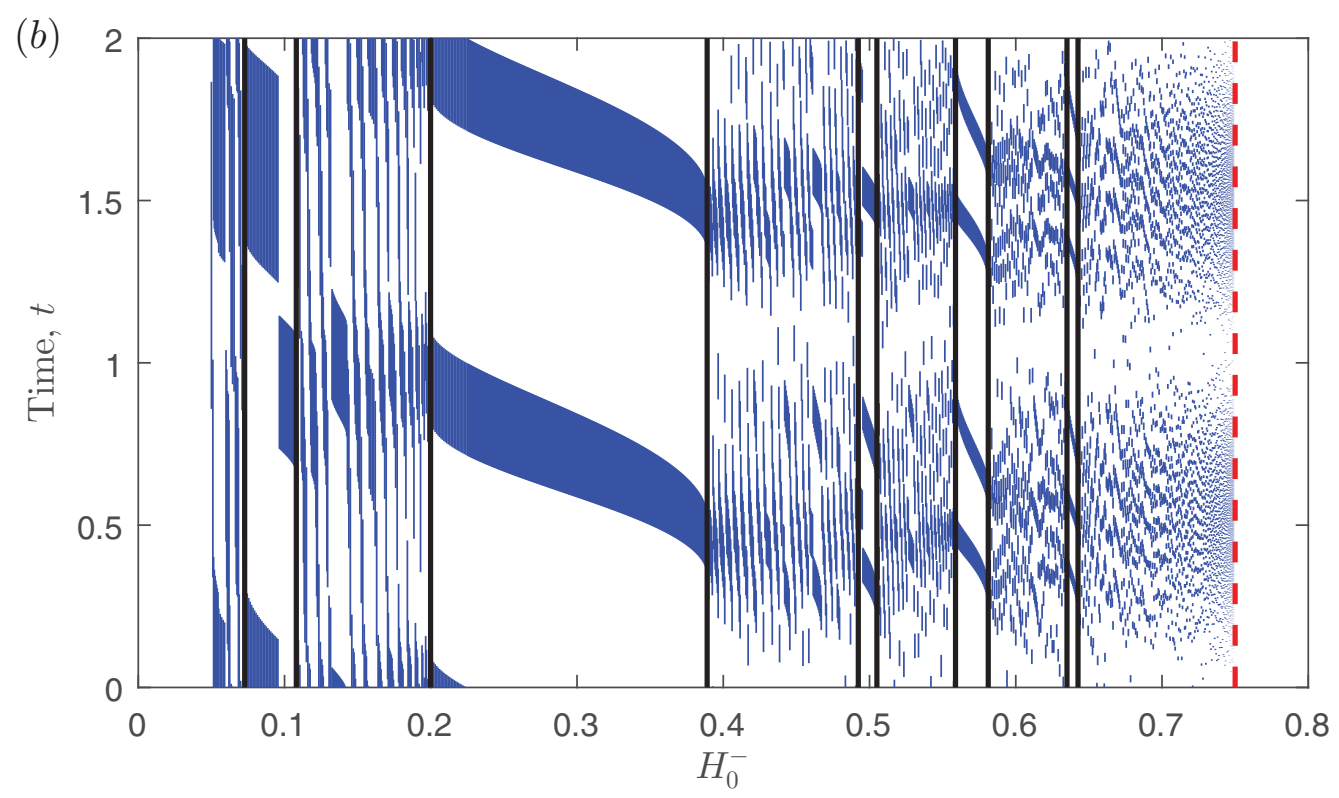

Figure 5. (a) Bifurcation set in the $\left(H_{0}^{-}, a\right)$-plane showing the largest few tongues where saddle-node bifurcations give the boundary of each tongue. (b) Bifurcation diagram for $a=0.05$. Sleep-wake patterns associated with the $\omega$-limit set, plotted over two days for a dense grid of $H_{0}^{-}$values, with sleep plotted in blue. Black lines in panel (a) and in panel (b) have been drawn to help identify the boundaries of the Arnold tongues at $a=0.05$, given by the dashed blue line in (a).

However, as will be discussed in the next two sections, this picture is altered for larger amplitudes.

To compute the bifurcation diagram, successive values of $H_{0}^{-}$have been taken. For each value, the two-process model has been iterated for 100 time units, which is sufficiently long for transients to decay, and then plotted. The vertical axis shows time over two time units with the blue regions representing times for which sleep occurs. Vertical black lines have been drawn to help guide the eye, and demark the regions of existence of the $(2,3),(1,1),(3,2),(2,1)$ and $(3,1)$ periodic solutions. The discontinuity in the band in the $(2,3)$ tongue might look surprising at first sight. However, this discontinuity represents the fact that for each $(2,3)$ periodic solution, there are two values on the upper threshold that can act as its starting point. The discontinuity is due to the numerical solution undergoing a phase shift between these starting points. 

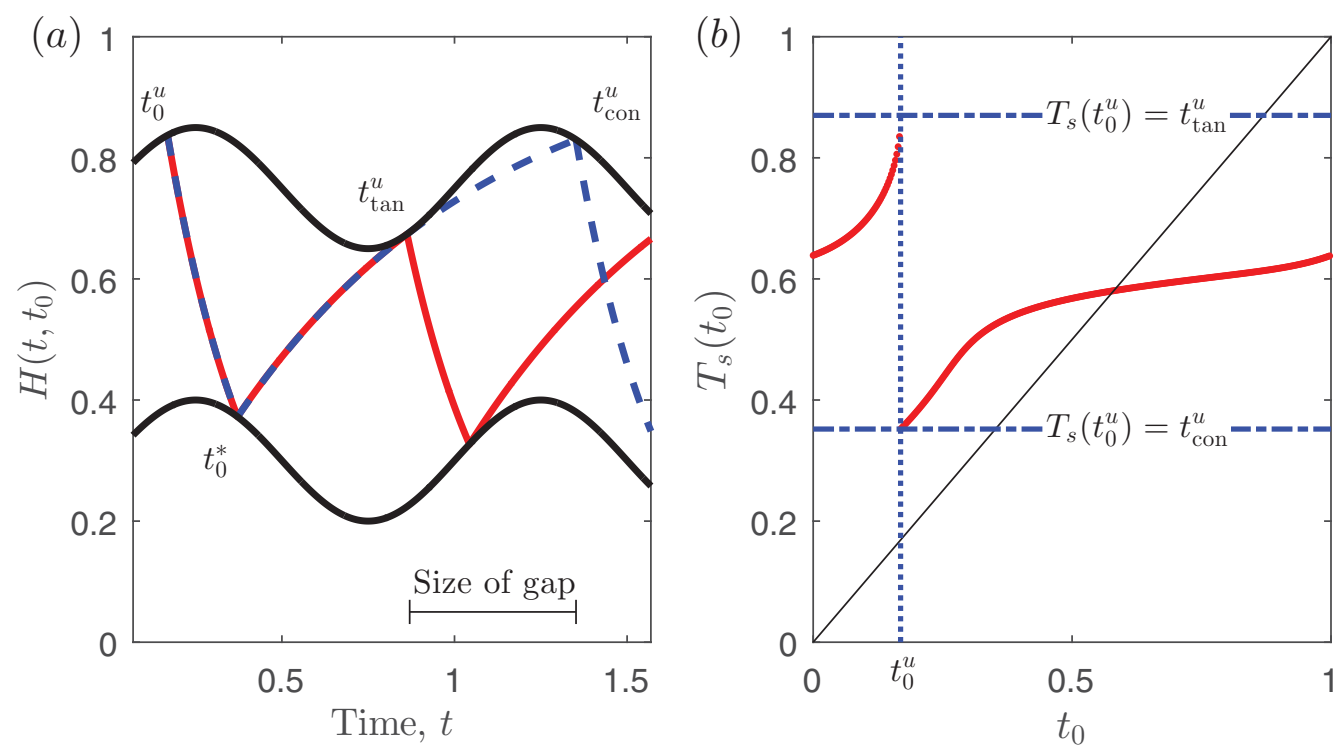

Figure 6. A small perturbation in start time $t_{0}$ can lead to large changes in the value of $T_{s}\left(t_{0}\right)$ if, for $t_{0}$, a tangency occurs between the homeostatic sleep pressure on sleep $H_{s}(t)$ and the lower threshold, or the homeostatic sleep pressure on wake $H_{w}(t)$ and the upper threshold. (a) Two possible trajectories with initial time $t_{0}^{u}$ are shown. The bold red line shows the homeostatic sleep pressure switching at the tangency point $H^{+}\left(t_{\text {tan }}^{u}\right)$, and dashed blue line shows the homeostatic sleep pressure missing the tangency and continuing on a wake trajectory. (b) The corresponding map shows the gap in $T_{s}\left(t_{0}\right)$ occurring at $t_{0}^{u}$. Here, we use $a=0.1$ and $H_{0}^{-}=0.3$.

\section{Gaps in the map}

In this section, we discuss the regions in the parameter plane for which the map is discontinuous (i.e., it has a gap), and the position and characteristics of this gap.

\subsection{Existence and structure of gaps}

As discussed in Skeldon et al. (2014) and Skeldon \& Derks (2017a), gaps in the map of the two-process model can arise when either the homeostatic sleep pressure during sleep, $H_{s}\left(t, t_{0}\right)$, becomes tangential to the lower threshold, $H^{-}(t)$, or the homeostatic sleep pressure during wake, $H_{w}\left(t, t_{0}\right)$, becomes tangential to the upper threshold, $H^{+}(t)$, as illustrated in Figure 6(a). The existence of gaps can in turn lead to border collisions, where a fixed point of the map collides with the gap.

In order to investigate the gaps, we consider first the case where $H_{s}\left(t, t_{0}\right)$ becomes tangential to the lower threshold, $H^{-}(t)$. Denoting the time a tangency occurs on the lower threshold as $t_{\tan }^{l} \equiv T_{d}\left(t_{0}^{l}\right)$, the conditions for the tangency to occur are that $H_{s}\left(t_{\tan }^{l}\right)=H^{-}\left(t_{\tan }^{l}\right)$ and that the gradients of the trajectory and the threshold match, $H_{s}^{\prime}\left(t_{\tan }^{l}\right)=\left(H^{-}\right)^{\prime}\left(t_{\tan }^{l}\right)$. Hence,

$$
H^{+}\left(t_{0}\right) e^{\frac{t_{0}-t_{\mathrm{tan}}^{l}}{\chi_{s}}}=H_{0}^{-}+a \sin \left(2 \pi t_{\mathrm{tan}}^{l}\right)
$$


and

$$
-\frac{H^{+}\left(t_{0}\right)}{\chi_{s}} e^{\frac{t_{0}-t_{\tan }^{l}}{\chi_{s}}}=2 \pi a \cos \left(2 \pi t_{\tan }^{l}\right)
$$

Substituting (4.1) into (4.2) and rearranging, it can be shown that $t_{\tan }^{l}$ satisfies

$$
\gamma_{s} \cos \left(2 \pi t_{\tan }^{l}+B\right)=-\frac{H_{0}^{-}}{a}
$$

where

and

$$
\gamma_{s}=\sqrt{4 \pi^{2} \chi_{s}^{2}+1}
$$

$$
B=2 \pi-\arccos \left(\frac{2 \pi \chi_{s}}{\gamma_{s}}\right)
$$

Note that $B \in\left(\frac{3 \pi}{2}, 2 \pi\right)$.

Solutions $t_{\tan }^{l}$ to (4.3) exist if

$$
-1 \leqslant \frac{H_{0}^{-}}{a \gamma_{s}} \leqslant 1
$$

The biological constraints (2.3) and the fact that $\gamma_{s}>1$ imply that the left-hand inequality is always satisfied, whereas the right-hand inequality gives a necessary condition on the circadian amplitude for the existence of gaps in the down map $T_{d}\left(t_{0}\right)$, namely,

$$
a \geqslant \frac{H_{0}^{-}}{\gamma_{s}}
$$

For $a>\frac{H_{0}^{-}}{\gamma_{s}},(4.3)$ has two possible solutions for $t_{\tan }^{l}$. These two solutions correspond to tangencies that would occur as a result of $H_{s}\left(t_{0}, t\right)$ approaching the lower threshold from above and from below, respectively. From the definition of the down map $T_{d}$, all $t \in\left(t_{0}, T_{d}\left(t_{0}\right)\right)$ have to satisfy $H_{s}\left(t_{0}, t\right) \geqslant H^{-}(t)$. Thus, only the tangency point $t_{\tan }^{l}$ that corresponds to the trajectory that approaches the lower threshold from above is relevant for gaps in the map. The local behaviour of $H_{S}$ near the tangency point can be found by considering the Taylor expansion of $H_{s}\left(t_{0}, t\right)-H^{-}(t)$ about the tangency point $t=t_{\tan }^{l}$. Since

$$
\begin{aligned}
H_{s}\left(t_{0}, t_{\tan }^{l}+\Delta t\right)-H^{-}\left(t_{\tan }^{l}+\Delta t\right) & =\left(H^{\prime \prime}\left(t_{\tan }^{l}\right)-H^{-^{\prime \prime}}\left(t_{\tan }^{l}\right)\right) \Delta t^{2}+\mathcal{O}\left(\Delta t^{3}\right) \\
& =\left(-\frac{2 \pi}{\chi_{s}} a \cos \left(2 \pi t_{\tan }^{l}\right)+4 \pi^{2} a \sin \left(2 \pi t_{\tan }^{l}\right)\right) \Delta t^{2}+\mathcal{O}\left(\Delta t^{3}\right),
\end{aligned}
$$

the right-hand side is greater than or equal to zero when $2 \pi \chi_{s} \sin \left(2 \pi t_{\tan }^{l}\right)-\cos \left(2 \pi t_{\tan }^{l}\right) \geqslant 0$. Using the definition of $B$ in (4.4), this can be written as $\gamma_{s} \sin \left(2 \pi t_{\tan }^{l}+B\right) \geqslant 0$; hence,

$$
0 \leqslant 2 \pi t_{\tan }^{l}+B \leqslant \pi
$$

Thus, (4.3) gives that a tangency point that is related to a gap in the map is given by

$$
2 \pi t_{\tan }^{l}+B=\arccos \left(-\frac{H_{0}^{-}}{a \gamma_{s}}\right) .
$$


Substituting the value of $B$ from equation (4.4) and rearranging give the time that a tangency occurs on the lower threshold, namely

$$
t_{\tan }^{l}=\frac{\arccos \left(-\frac{H_{0}^{-}}{a \gamma_{s}}\right)+\arccos \left(\frac{2 \pi \gamma_{s}}{\gamma_{s}}\right)}{2 \pi},
$$

for $-a<H_{0}^{-} \leqslant a \gamma_{s}$. Since the lower threshold has to have a negative gradient to be tangential to the homeostatic sleep pressure on sleep, the value of $t_{\text {tan }}^{l}$ lies in the region $\left(\frac{1}{4}, \frac{3}{4}\right)$.

Having identified the tangency point related to a gap, we next look at the point $t_{0}^{l}$ that is mapped by $T_{d}$ into $t_{\mathrm{tan}}^{l}$, that is, the point $t_{0}^{l}$ that satisfies $T_{d}\left(t_{0}^{l}\right)=t_{\mathrm{tan}}^{l}$. By rearranging the tangency condition (4.2), it can be seen that $t_{0}^{l}$ satisfies

$$
\left(H_{0}^{+}+a \sin \left(2 \pi t_{0}^{l}\right)\right) \exp \left(\frac{t_{0}^{l}}{\chi_{s}}\right)=-2 \pi \chi_{s} a \cos \left(2 \pi t_{\tan }^{l}\right) \exp \left(\frac{t_{\tan }^{l}}{\chi_{s}}\right) .
$$

This is an implicit equation that can be solved numerically. To summarise, to find the position of the gap in the map $t_{0}^{l}$, first $t_{\tan }^{l}$ is found from equation (4.7), and then equation (4.8) is solved numerically to find $t_{0}^{l}$, where $t_{0}^{l}<t_{\text {tan }}^{l}$.

The second mechanism to create gaps is via $H_{w}\left(t_{0}, t\right)$ becoming tangent to the upper threshold $H_{0}^{+}$. These tangencies lead to gaps in the up map, $T_{u}\left(t_{0}\right)$. A discrete symmetry exists in the two-process model that maps the solution for the sleep and wake trajectories onto each other. The sleep trajectory is mapped into a wake trajectory by using the mapping

$$
\left(t, H_{s}, \chi_{s}, H_{0}^{-}, H_{0}^{+}, a, t_{0}\right) \mapsto\left(t+\frac{1}{2}, 1-H_{w}, \chi_{w}, 1-H_{0}^{+}, 1-H_{0}^{-}, a, t_{0}+\frac{1}{2}\right) .
$$

This symmetry allows us to directly state the criteria for tangencies of the wake homeostatic pressure with the upper threshold. Specifically, applying the mapping to (4.5) gives that the homeostatic sleep pressure on wake has a tangency (and hence the up map $T_{d}$ has a gap) if

$$
a \geqslant \frac{1-H_{0}^{+}}{\gamma_{w}}
$$

where $\gamma_{w}=\sqrt{4 \pi^{2} \chi_{w}^{2}+1}$. The tangency from below is at

$$
t_{\tan }^{u}=\frac{\arccos \left(\frac{2 \pi \chi_{w}}{\gamma_{w}}\right)-\arccos \left(\frac{1-H_{0}^{+}}{a \gamma_{w}}\right)}{2 \pi} .
$$

The initial time $t_{0}^{*}$ on the lower threshold that leads to this tangency satisfies

$$
\left(1-H_{0}^{-}-a \sin \left(2 \pi t_{0}^{*}\right)\right) \exp \left(\frac{t_{0}^{*}}{\chi_{w}}\right)=2 \pi a \chi_{w} \cos \left(2 \pi t_{\tan }^{u}\right) \exp \left(\frac{t_{\tan }^{u}}{\chi_{w}}\right) .
$$

Thus, the up map $T_{u}(t)$ has a gap at $t=t_{0}^{*}$. As $T_{s}=T_{u} \circ T_{d}$, this leads to a gap in the sleep map $T_{s}$ only if $t_{0}^{*}$ lies in the range of $T_{d}$. If $t_{0}^{*}$ is in the range of $T_{d}$, then the initial time $t_{0}^{u}$ on the upper threshold, which leads to the tangency time $t_{\tan }^{u}$ (i.e., $\left.T_{s}\left(t_{0}^{u}\right)=t_{\tan }^{u}\right)$, is 
characterised by

$$
H^{-}\left(t_{0}^{*}\right) \exp \left(\frac{t_{0}^{*}}{\chi_{s}}\right)=H^{+}\left(t_{0}^{u}\right) \exp \left(\frac{t_{0}^{u}}{\chi_{s}}\right) .
$$

In Figure 6, the values $t_{0}^{u}$ and $t_{0}^{*}$ are labelled to help visualise this process. The parameter values in Figure 6 are chosen such that the up map $T_{u}$ has a gap, but the down map $T_{d}$ has no gap.

Having established when and where tangencies occur on the lower and upper threshold and how this introduces gaps, we can now focus on characterising the other endpoint of the gap as this will be needed for the derivation of the border collisions.

\subsection{Characterising the gap}

By continuing the homeostatic sleep pressure on sleep $H_{S}$ through the tangency point, $H^{-}\left(t_{\tan }^{l}\right)$, until the next hit on the lower threshold, the other endpoint of the gap in the down map $T_{d}\left(t_{0}\right)$ can be found. Denoting this endpoint found by continuation by $t_{\text {con }}^{l}$, it can be characterised as the first $t_{\mathrm{con}}^{l}>t_{\mathrm{tan}}^{l}$, which satisfies

$$
H^{-}\left(t_{\tan }^{l}\right) e^{\frac{t_{\tan }^{l}-t_{\mathrm{con}}^{l}}{\chi_{s}}}=H^{-}\left(t_{\mathrm{con}}^{l}\right)
$$

By rearranging (4.13), we get

$$
H^{-}\left(t_{\mathrm{con}}^{l}\right) e^{\frac{t_{\mathrm{con}}^{l}}{\chi_{s}}}=H^{-}\left(t_{\mathrm{tan}}^{l}\right) e^{\frac{t_{\mathrm{tan}}^{l}}{\chi_{s}}}, \quad t_{\mathrm{con}}^{l}>t_{\mathrm{tan}}^{l},
$$

which can be solved implicitly giving the size of the gap in $T_{d}$ as $t_{\text {con }}^{l}-t_{\tan }^{l}$. Since $T_{s}=T_{u} \circ T_{d}$, the size of the gap in $T_{s}$ is $T_{u}\left(t_{\mathrm{con}}^{l}\right)-T_{u}\left(t_{\mathrm{tan}}^{l}\right)$.

The same approach can be used for tangencies on the upper threshold, giving the implicit definition

$$
\left(1-H^{+}\left(t_{\mathrm{con}}^{u}\right)\right) e^{\frac{t_{\mathrm{con}}^{u}}{\alpha_{w}}}=\left(1-H^{+}\left(t_{\mathrm{tan}}^{u}\right)\right) e^{\frac{t_{\mathrm{tan}}^{u}}{\alpha_{w}}}, \quad t_{\mathrm{con}}^{u}>t_{\mathrm{tan}}^{u}
$$

By continuing the homeostatic sleep pressure on wake through the tangency point, $t_{\tan }^{u}$, until the next hit on the upper threshold $t_{\mathrm{con}}^{u}$, one finds that the size of the gap in the up map $T_{u}$ is given by $t_{\text {con }}^{u}-t_{\text {tan }}^{u}$. If the down map $T_{d}$ has no discontinuities, then this is also the size of the gap in $T_{s}$. If the down map $T_{d}$ has discontinuities and $t_{0}^{*}$ is in the range of $T_{d}$, then this is the size of a second gap in $T_{s}$, otherwise $T_{s}$ has no second gap. In Figure 6, we show the relationship between the two-process model and the size of the gap, illustrating that the size of the gap is the same in $T_{u}$ as in $T_{s}$. The parameters are such that $T_{u}$ has a gap, but $T_{d}$ is continuous.

As can be seen in Figures 2 and 6, the derivative of the map to the left of the gap in the down, up and sleep maps appears to be infinite. This is indeed an intrinsic feature of maps generated from the two-process model, which can be seen from the expressions for the derivatives of the maps. Differentiating the expression for the sleep map $T_{s}(2.5)$ with respect to $t_{0}$ gives

$$
T_{s}^{\prime}\left(t_{0}\right)=T_{u}^{\prime}\left(T_{d}\left(t_{0}\right)\right) T_{d}^{\prime}\left(t_{0}\right)
$$


When $T_{d}\left(t_{0}\right)$ and $T_{u}\left(T_{d}\left(t_{0}\right)\right)$ are locally well defined, differentiation of the implicit relations for $T_{d}$ and $T_{u}$ in (2.4) with respect to $t_{0}$ and use of the explicit definitions of the sleep and wake homeostatic sleep pressure in (2.1) shows that

$$
T_{d}^{\prime}\left(t_{0}\right)=\frac{\left(2 \pi a \cos \left(2 \pi t_{0}\right)+\frac{H_{0}^{+}}{\chi_{s}}+\frac{a}{\chi_{s}} \sin \left(2 \pi t_{0}\right)\right) e^{\frac{t_{0}-T_{d}\left(t_{0}\right)}{\chi_{s}}}}{\left(2 \pi a \cos \left(2 \pi T_{d}\left(t_{0}\right)\right)+\frac{H_{0}^{-}}{\chi_{s}}+\frac{a}{\chi_{s}} \sin \left(2 \pi T_{d}\left(t_{0}\right)\right)\right)}
$$

and

$$
T_{u}^{\prime}\left(T_{d}\right)=\frac{\left(2 \pi a \cos \left(2 \pi T_{d}\right)+\frac{H_{0}^{-}-1}{\chi_{w}}+\frac{a}{\chi_{w}} \sin \left(2 \pi T_{d}\right)\right) e^{\frac{T_{d}\left(t_{0}\right)-T_{u}\left(T_{d}\right)}{\chi_{w}}}}{\left(2 \pi a \cos \left(2 \pi T_{u}\left(T_{d}\right)\right)+\frac{H_{0}^{+}-1}{\chi_{w}}+\frac{a}{\chi_{w}} \sin \left(2 \pi T_{u}\left(T_{d}\right)\right)\right)} .
$$

Furthermore, combining the tangency conditions (4.1) and (4.2) gives that a tangency point $t_{\tan }^{l}$ satisfies $2 \pi a \cos \left(2 \pi t_{\tan }^{l}\right)+\frac{H_{0}^{-}}{\chi_{s}}+\frac{a}{\gamma_{s}} \sin \left(2 \pi t_{\tan }^{l}\right)=0$. Thus, if $t_{0}^{l}$ is such that $T_{d}\left(t_{0}^{l}\right)=$ $t_{\tan }^{l}$, then (4.17) shows that $T_{d}$ is not differentiable at $t_{0}^{l}$ and $T_{d}^{\prime}\left(t_{0}\right) \rightarrow \infty$ as $t_{0}$ approaches $t_{0}^{l}$ from the left. As $t_{0}$ approaches $t_{0}^{l}$ from the right, then $T_{d}\left(t_{0}\right)$ approaches to $t_{\text {con }}^{l}$ and (4.17) shows that the derivative is finite. Similarly, if $t_{0}^{u}$ is such that $T_{u}\left(T_{d}\left(t_{0}^{u}\right)\right)=t_{\tan }^{u}$, then $\left.T_{u}^{\prime}\left(T_{d}\left(t_{0}^{u}\right)\right)\right) \rightarrow \infty$ as $t_{0}$ approaches $t_{0}^{u}$ from the left and is finite if $t_{0}$ approaches $t_{0}^{u}$ from the right.

We note that the $t$-interval in which the gradient of the map increases towards infinity can be very small, so in a numerical simulation study it is easy to miss the fact that the gradient becomes infinite. Much of the literature on maps with gaps (see, for example, the review paper by Granados et al., 2017) focuses on piecewise linear maps where the derivative is necessarily finite on both sides, although Pring \& Budd (2011) note that infinite derivatives at a gap arise naturally in many applications.

\subsection{Monotonicity}

Just as a gap is created in the down map $T_{d}$ when there is a tangency between the homeostatic sleep pressure during sleep and the lower threshold $\mathrm{H}^{-}$, it has a turning point (and hence is non-monotonic) when there is a tangency between the homeostatic sleep pressure during sleep and the lower threshold $\mathrm{H}^{-}$. This mechanism for the development of non-monotonicity is discussed in more detail by Skeldon \& Derks (2017a).

As the upper threshold and the lower threshold are identical apart from in their mean value, the work from Section 4.1 can be used to derive the criteria for non-monotonicity of the down, up and sleep maps. So, for the down map, we get from (4.5), with the mean value $H_{0}^{-}$replaced by the mean value $H_{0}^{+}$, that the down map $T_{d}$ may be non-monotonic if

$$
a>\frac{H_{0}^{+}}{\gamma_{s}} .
$$

For the up map, we get from (4.9), with the mean value $H_{0}^{+}$replaced by the mean value $H_{0}^{-}$, that the up map $T_{u}$ is non-monotonic if

$$
a>\frac{1-H_{0}^{-}}{\gamma_{w}} .
$$


Thus, the sleep map $T_{s}=T_{d}\left(T_{u}\right)$ may be non-monotonic if

$$
a>\min \left(\frac{H_{0}^{+}}{\gamma_{s}}, \frac{1-H_{0}^{-}}{\gamma_{w}}\right) .
$$

\section{Border collisions}

The presence of gaps in the map can alter the transitory behaviour, but more significantly gives an alternative mechanism for the creation/destruction of fixed points of the map and thus periodic solutions in the two-process model, via border collisions. At a border collision, a fixed point of the map (or its iterate) coincides with the endpoint of a gap. Two types of border collision are possible within the two-process model. First, where a fixed point of the map coincides with the side of the gap where the derivative of the map is infinite, resulting in the creation/destruction of an unstable fixed point. In what follows, these will be referred to in this paper as Type I border collisions. Second, where a fixed point of the map coincides with the side of the gap where the derivative is finite, resulting in the creation/destruction of either a stable or an unstable fixed point. These will be referred to in this paper as Type II border collisions. We distinguish between the two types of border collisions as a saddle-node bifurcation can collide with a finite derivative border collision (if the derivative equals 1), but it can never collide with an infinite derivative border collision. These two types of border collision are illustrated in Figure 7.

Clearly, since gaps can result from tangencies with either the lower threshold or upper threshold, four cases should be considered, namely Type I and II border collisions for the lower threshold and Type I and II border collisions for the upper threshold. In the next two subsections, we focus on methods for computing border collisions with gaps due to tangencies occurring on the upper threshold, but similar ideas can be used to find those due to the lower threshold.

\subsection{Computation of Type I border collisions}

To find Type I border collisions of $(p, q)$ periodic solutions numerically, we fix all parameters except for $H_{0}^{-}$. The Type I border collision with a gap due a tangency with the upper threshold will occur when there is a $(p, q)$ periodic solution at $t_{\tan }^{u}$. We use the explicit expression from equation (4.10) to find the tangency time $t_{\tan }^{u}$. We then take an initial guess for the parameter $H_{0}^{-}$and use a non-linear solver to iterate on this parameter to find the value of $H_{0}^{-}$such that the forward iteration of the two-process model starting on the upper threshold at $t=t_{\tan }^{u}$ and following the homeostatic sleep pressure until $t=t_{\tan }^{u}+q$ leads to a return to the appropriate value on the upper threshold. In Figure 7(a) a typical example of a $(1,1)$ periodic sleep-wake cycle undergoing a Type I border collision is shown. Figure 7(b) shows the associated one-dimensional map, clearly showing the infinite derivative at the border collision.

\subsection{Computation of Type II border collisions}

A similar idea can be used for the computation of Type II border collisions of $(p, q)$ periodic solutions. A Type II border collision due a tangency with the upper threshold 

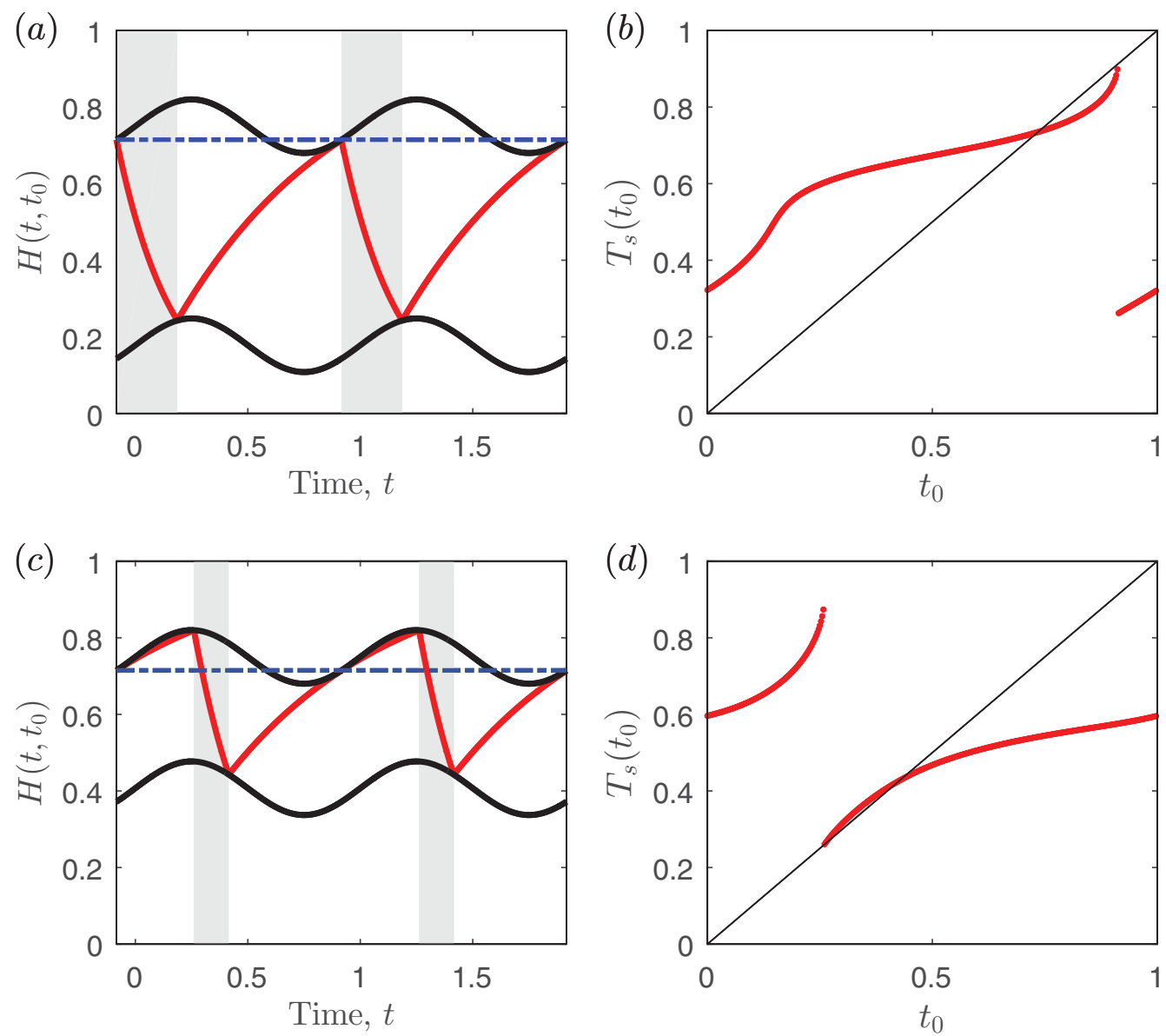

Figure 7. Type I and Type II border collisions of a $(1,1)$ periodic solution are shown for $a=0.07$. The dashed blue lines in (a) and (c) represent the value, $H^{+}\left(t_{\text {tan }}^{u}\right)$. (a) A sleep-wake cycle for a Type I border collision, switching from wake to sleep occurs at the time $t_{\tan }^{u}$ on the upper threshold. (b) The corresponding one-dimensional map shows the infinite derivative at the Type I border collision. (c) A sleep-wake cycle for a Type II border collision where the homeostatic sleep pressure on wake misses the upper threshold at $H^{+}\left(t_{\text {tan }}^{u}\right)$, switching at a later time (see Section 4.2). (d) The one-dimensional map for the Type II border collision. In (a) and (b), we have $H_{0}^{-}=0.178$, and for (c) and (d) $H_{0}^{-}=0.4071$.

will occur when there is $(p, q)$ periodic orbit at $t_{\text {con }}^{u}$. Equation (4.10) gives the tangency time $t_{\tan }^{u}$ and the time point at the other side of the gap $t_{\text {con }}^{u}$ is found by solving the implicit relation in (4.15). The forward iteration starts at this point and $\mathrm{H}_{0}^{-}$is tuned such that the forward iteration of the two-process model starting on the upper threshold at $t=t_{\text {con }}^{u}$ and following the homeostatic sleep pressure until $t=t_{\text {con }}^{u}+q$ leads to a return to the appropriate value on the upper threshold. In Figure 7(c), we show how $t_{\text {con }}^{u}$ is found by continuing the homeostatic sleep pressure on a wake trajectory from the tangency $t_{\text {tan }}^{u}$ and the resulting $(1,1)$ periodic solution for the tuned $H_{0}^{-}$value. The associated map in 
Figure 7(d) shows that the fixed point now coincides with the other side of the gap where the derivative is finite.

\subsection{Bifurcation set: Saddle-node tongues and border collisions}

In this section, we illustrate how gaps in the maps change the bifurcation set: one of the two saddle-node bifurcation boundaries is replaced by a border collision boundary and the border collisions inside the tongue decrease the number of periodic solutions to one periodic solution. Figure 8 shows the position of the two types of border collisions and the saddle-node bifurcations in the $\left(H_{0}^{-}, a\right)$ parameter plane. From the analysis in Section 4.1, it follows that for $a<\frac{H_{0}^{-}}{\gamma_{s}}$ and $a<\frac{1-H_{0}^{+}}{\gamma_{w}}$, there are no tangencies in the two-process model and consequently no gaps in the one-dimensional map. In this region, the one-dimensional map is the lift of a continuous circle map and regions of $(p, q)$ periodic solutions form Arnold tongues bordered by saddle-node bifurcations, where within each tongue there is a pair of periodic solutions, one stable and one unstable.

As discussed in Section 4.1, for $a \geqslant \frac{H_{0}^{-}}{\gamma_{s}}$ or $a \geqslant \frac{1-H_{0}^{+}}{\gamma_{w}}$, gaps can occur in the map $T_{s}$. For some regions, the presence of gaps has no impact on the number and stability of fixed points of the map, although their presence can alter the transient dynamical behaviour. However, as can be seen in Figure 8, the gaps lead to border collisions that define roughly u-shaped regions 'in' each tongue. The left-hand side of these u-shaped regions are linked to Type I border collisions and appear to asymptote to the saddle-node bifurcation line. Note that these two types of bifurcations cannot coincide because at the saddle-node bifurcation the gradient of the map is one, whereas at the Type I border collision the gradient of the map is infinite.

The right-hand side of $\mathrm{u}$-shaped region is linked to Type II border collisions. The saddle-node bifurcation and the Type II border collision meet at a point where the gradient of the map at the Type II border collision is unity, leaving only the border collision. This explains how the right saddle-node curves in Figure 5 terminate: they are replaced by the border collision curve.

Within the u-shaped region, there is only one periodic solution. For the parameter values we have explored, the map is monotonic and has a unique rotation number $\frac{q}{p}$ corresponding to the $(\mathrm{p}, \mathrm{q})$ periodic orbits. The Type I border collision removes/adds an unstable periodic solution (due to its infinite derivative); hence, the remaining solution is stable.

For the $\left(H_{0}^{+}, \chi_{s}, \chi_{w}\right)$ parameter choice shown in Figure 8, the Arnold saddle-node tongues with rotation number $\frac{q}{p}<1$ end in the region with $\frac{1-H_{0}^{+}}{\gamma_{w}}<a<\frac{H_{0}^{-}}{\gamma_{s}}$. In this region, the up map $T_{u}$ has a gap, but the down map $T_{d}$ does not have a gap. Thus the relevant border collisions for these tongues are those due to a tangency with the upper threshold. The right endpoint of the $(1,1)$ tongue is in this region as well; hence, here, the border collision due to a tangency with the upper threshold is relevant. The left endpoint of the $(1,1)$ tongue is in the region with $a>\max \left(\frac{H_{0}^{-}}{\gamma_{s}}, \frac{1-H_{0}^{+}}{\gamma_{w}}\right)$, hence both border collisions due a tangency with the upper threshold and due to a tangency with the lower threshold could play a role. For the parameter values used in Figure 8, we have not found any numerical evidence for border collisions due to the lower thresholds in the $(1,1)$ tongue. 


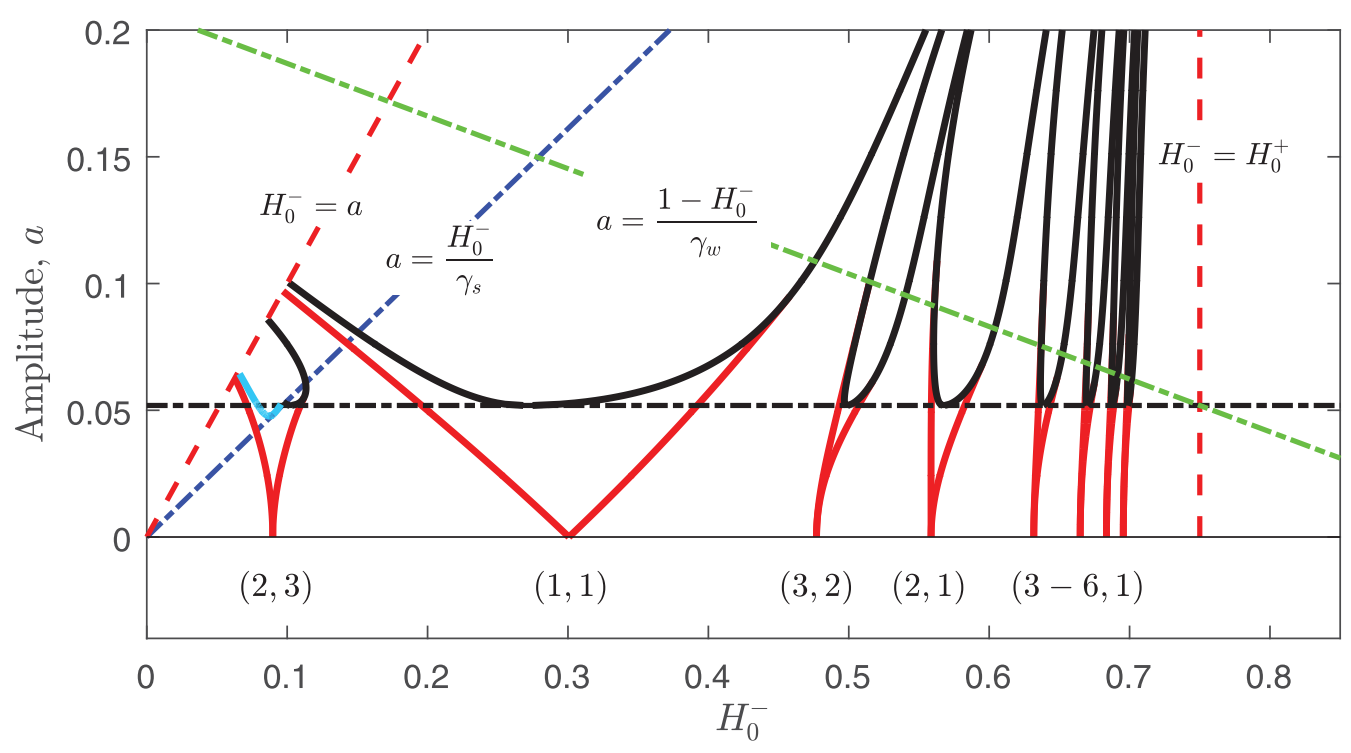

Figure 8. Bifurcation set in the $\left(H_{0}^{-}, a\right)$-plane. Tangencies on the upper threshold occur above the dot-dashed black line corresponding to $a=\frac{1-H_{0}^{+}}{\gamma_{w}}$. Tangencies on the lower threshold occur above the dot-dashed blue line. As in Figure 5, the saddle-node bifurcations are shown as solid red lines. Border collisions emerge from the lines demarking the onset of tangencies, forming u-shaped regions. These are shown in black for those occurring as a result of tangency with the upper threshold and in light blue for those resulting from tangency with the lower threshold. Type I border collisions occur to the left of each u-shaped region and Type II border collisions occur to the right. The bifurcations are bounded by the biological constraints on the parameters (2.3) (dashed red). Above the dot-dashed green line, the map may be non-monotonic.

The tongues with rotation number $\frac{q}{p}>1$ end in regions where border collisions with both the upper and lower thresholds potentially are important. In the $(2,3)$ tongue, it can be seen how the curve for the Type II border collision with the lower threshold intersects with the curve for the Type I border collision with the upper threshold. At this point in parameter space, the endpoint of the gap due to the lower threshold, $t_{\text {con }}^{l}$, coincides with the start point of the gap due to the upper threshold, $t_{\tan }^{u}$. Hence, the endpoint of gap due to the lower threshold 'jumps' to $t_{\text {con }}^{u}$. This is an illustration of how the tangency on the upper threshold loses relevance, see the discussion under (4.11) for more details. As illustrated by the plot, at the intersection of the two curves, the border collisions disappear.

\section{Conclusions}

In this paper, we have discussed an interesting non-smooth dynamical system that arises in the context of sleep/wake regulation. This system brings together results from continuous monotonic circle maps and maps with gaps, resulting in a novel bifurcation set in which both border collisions and saddle-node bifurcations create/destroy fixed points of the map. The fixed points in the map correspond to periodic solutions in the full dynamical system and represent different (periodic) patterns of sleep/wake. We see that at low circadian 
amplitudes, varying the bifurcation parameter $H_{0}^{-}$leads to a sequence of periodic solutions arising through saddle-node bifurcations, as is known to happen in continuous monotonic circle maps. For fixed $a$ and varying $H_{0}^{-}$, the rotation number associated with the map has a devil's staircase structure and the intervals with constant rotation number are bounded by saddle-node bifurcations. At larger amplitudes, varying the same parameter leads to the same devil's staircase sequence for the rotation number, but now both border collisions and saddle-node bifurcations are important for the creation/destruction of periodic orbits.

In Section 4.3, we have seen that, for larger values of $a$, the maps $T_{d}, T_{u}$ and $T_{s}=T_{u} \circ T_{d}$ are not monotonic. We note that non-monotonicity has the potential to lead to more complex dynamics, including period-doubling and chaos, as was shown for continuous but non-invertible maps of the circle by Mackay \& Tresser (1986). In the results presented here, we focused on monotonic maps. A more extensive discussion of the consequences of non-monotonicity is in Derks et al. (2018) for a simplified example that contains the key features of the two-process model.

Within the context of sleep/wake regulation, understanding the dynamics of the twoprocess model is important for two reasons. First, because the two-process model is very influential within sleep and circadian science and second, because it remains at the heart of more complicated models of sleep. Many of the numerical results found in these more complicated models can be understood by understanding the dynamics of the two-process model.

The different patterns of sleep that arise in the model have biological relevance. Although a common pattern of human sleep corresponds to one sleep a day, $(p, q)=(1,1)$, referred to as monophasic sleep, other patterns of sleep do occur. For humans, in some cultures, the usual practice is to take a siesta, see Barone (2000); Ekirch (2005) suggests that two sleeps at night, with only a short wake period between the two, was the historical norm in some societies. This suggests that relatively small sociological or physiological changes could result in a different sleep pattern.

Patterns of sleep with more than one sleep a day are referred to as polyphasic sleep. In babies and pre-school children, there is a gradual transition from polyphasic to monophasic sleep, see Galland et al. (2012). In Skeldon et al. (2014), it was suggested that these could plausibly be explained by gradual changes in physiological parameters that result in slowly varying parameters. Within the context of this paper, this would correspond to a gradual increase in the natural period $T_{\text {nat }}$ of the homeostatic sleep system resulting in a transition across the tongues corresponding to decreasing numbers of sleep episodes a day.

Transitions between different kinds of periodic solutions are also relevant in experimental studies showing internal desynchrony. When humans are isolated from external time cues, a circadian rhythm remains, but is no longer entrained to $24 \mathrm{~h}$ : for most humans, the circadian rhythm is a little longer than 24 h, see Aschoff (1965). Patterns of sleep/wake then: either alter to remain in synchrony with the adjusted circadian period, but with an altered phase relationship because of the change in period or desynchronise, or switch to a rhythm of one sleep every two days, a so-call circabidian rhythm, see Wever (1979). We have scaled time so that the circadian period is one, so a lengthened circadian period in the original system is equivalent to increasing $T_{\text {nat }}$ as occurs by decreasing $H_{0}^{-}$ or increasing $H_{0}^{+}, \chi_{s}$ or $\chi_{w}$. The switch to circabidian rhythms would therefore correspond 
to a transition from the $(p, q)=(1,1)$ tongue to the $(1,2)$ tongue. This structure gives the theoretical underpinning for the results of the numerical study carried out in Phillips et al. (2011a) to investigate internal desynchrony and bicircabidian rhythms in a neuronal model.

This same Arnold tongue framework also explains the polyphasic patterns seen in Phillips et al. (2010) used to describe different patterns of mammalian sleep and the original results of Daan et al. (1984). We note that in Daan et al. (1984) the circadian oscillation includes not only a $\sin 2 \pi t$ term but also a small amplitude higher harmonic term, $\sin 6 \pi t$. We would not expect this to change the qualitative Arnold tongue/border collision structure, as the higher order terms are a small perturbation; hence, the main features of monotonicity together with a transition to maps with gaps is preserved.

We note that the differences in transient dynamical behaviour that result from the presence of gaps can also have biological relevance, as is discussed by Booth et al. (2017) who consider the construction of one-dimensional maps in a neuronal model that models wake and two stages of sleep (rapid eye movement and non-rapid eye movement). This neuronal model also has the two-process model at its heart, although the additional sleep stage results in additional gaps.

Finally, we note that although there is an extensive literature on maps, previous studies have either focussed on maps with gaps or maps with no gaps. This is the first example of which we are aware where the transition between the two is considered. The results are illuminating, showing how border collision can take over from saddle-node bifurcations to form boundaries of Arnold tongues and highlighting a link between the devil's staircase structure of periodic solutions seen in both cases.

\section{Acknowledgements}

The authors are indebted to Prof. Derk-Jan Dijk of the Surrey Sleep Research Centre (University of Surrey) for introducing them to the area of sleep-wake regulation. The authors also acknowledge helpful discussions with Prof. Paul Glendinning (University of Manchester) and discussions with participants at the intensive programme on Advances in Nonsmooth Dynamics at the Centre de Recerca Matemática in Spring 2016.

\section{References}

Archer, S. N., Laing, E. E., Möller-Levet, C. S., van der Veen, D. R, Bucca, G., Lazar, A. S., Santhi, N., Slak, A., Kabiljo, R., von Schantz, M., Smith, C. P. \& Dijk, D.-J. (2014) Mistimed sleep disrupts circadian regulation of the human transcriptome. Proc. Natl. Acad. Sci. USA 111, E682-E691.

ARnold, V. I. (1991) Cardiac arrhythmias and circle mappings. Chaos 1, 20-21, reprinted from Arnold's thesis (Moscow 1959).

Aschoff, J. (1965) Circadian rhythms in man. Science 148, 1427-1432.

Avrutin, V., Schantz, M. \& BanerJee, S. (2006) Multi-parametric bifurcations in a piecewise-linear discontinuous map. Nonlinearity 19, 1875-1906.

Barone, T. L. (2000) Is the siesta an adaptation to disease? Hum. Nat. 11, 233-258.

Booth, V. \& Diniz Behn, C. G. (2014) Physiologically-based modeling of sleep-wake regulatory networks. Math. Biosci. 250, 54-68. 
Booth, V., Xique, I. \& Diniz Behn, C. G. (2017) One-dimensional map for the circadian modulation of sleep in a sleep-wake regulatory network model for human sleep. SIAM J. Appl. Dyn. Syst. 16, 1089-1112.

Borbély, A. A. (1982) A two-process model of sleep regulation. Hum. Neurobiol. 1, 195-204.

DaAn, S., Beersma, D. G. M. \& BorbéLy, A. A. (1984) Timing of human sleep: Recovery process gated by a circadian pacemaker. Am. J. Physiol. 246, R161-R178.

Derks, G., Glendinning, P. A. \& Skeldon, A. C. (2018) Transitions to gaps and non-monotonicity in circle maps. In preparation.

Diniz Behn, C. G. \& Bоотн, V. (2010) Simulating microinjection experiments in a novel model of the rat sleep-wake regulatory network. J. Neurophysiol. 103, 1937-1953.

Diniz Behn, C. G., Brown, E. N., Scammell, T. E. \& Kopell, N. J. (2007) Mathematical model of network dynamics governing mouse sleep-wake behaviour. J. Neurophysiol. 97, 3828-3840.

Ekirch, A. R. (2005) At Day's Close: A History of Nighttime. W.W. Norton \& Company, New York.

Galland, B. C., Taylor, B. J., Elder, D. E. \& Herbison, P. (2012) Normal sleep patterns in infants and children: A systematic review of observational studies. Sleep Med. Rev. 16, 213-222.

Granados, A., Alsedì, L. \& Krupa, M. (2017) The period adding and incrementing bifurcations: From rotation theory to applications. SIAM Rev. 59, 225-292.

Keener, J. P. (1980) Chaotic behaviours in piecewise continuous difference equations. Trans. Am. Math. Soc. 261, 589-604.

KNUTSON, K. L. (2010) Sleep duration and cardiometabolic risk: A review of the epidemiologic evidence. Best Pract. Res.: Clin. Endocrinol. Metab. 24, 731-743.

Kumar, R., Bose, A. \& Mallick, B. N. (2012) A mathematical model towards understanding the mechanism of neuronal regulation of wake-NREMS-REMS states. PLoS One 7, e42059.

Luyster, F. S., Strollo, P. J. JR., Zee., P. C. \& Walsh, J. K. (2012) Sleep: A health imperative. Sleep 35, 727-734.

Mackay, R. \& Tresser, C. (1986) Transition to topological chaos for circle maps. Physica D 19, 206-237.

Möller-Levet, C. S., Archer, S. N., Bucca, G., Laing, E., Slak, A., Kabiljo, R., Lo, J., Santhi, N., von Schantz, M., Smitth, C. P. \& DiJk, D.-J. (2013) Effects of insufficient sleep on circadian rhythmicity and expression amplitude of the human blood transcriptome. Proc. Natl. Acad. Sci. USA 110, E1132-E1141.

Nakao, M., Sakai, H. \& Yamamoto, M. (1997) An interpretation of the internal desynchronisations based on dynamics of the two-process model. Methods Inform. Med. 36(4-5), 282-285.

NakaO, M. \& Yamamoto, M. (1998) Bifurcation properties of the two-process model. Psychiatry Clin. Neurosci. 52, 131-133.

Nielsen, L. S., Danielsen, K. \& Sorensen, T. (2011) Short sleep duration as a possible cause of obesity: Critical analysis of the epidemiological evidence. Obes. Rev. 12, 78-92.

Phillips, A. J. K., Czeisler, C. A. \& Klerman, E. B. (2011 Revisiting spontaneous internal desynchrony using a quantitative model of sleep physiology. J. Biol. Rhythms 26, 441-453.

Phillips, A. J. K. \& Robinson, P. A. (2007) A quantitative model of sleep-wake dynamics based on the physiology of the brainstem ascending arousal system. J. Biol. Rhythms 22, 167-179.

Phillips, A. J. K., Robinson, P. A., Kedziora, D. J. \& Abeysuriya, R. G. (2010) Mammalian sleep dynamics: How diverse features arise from a common physiological framework. PLoS Comput. Biol. 6, e1000826.

Postnova, S., Voigt, K. \& Braun, H. (2009) A mathematical model of homeostatic regulation of sleep-wake cycles by hypocretin/orexin. J. Biol. Rhythms 24(6), 523-535.

Pring, S. R. \& BUdD, C. J. (2011) The dynamics of a simplified pin-ball machine. IMA J. Appl. Math. 76, 67-84.

Rempe, M. J., Best, J. \& Terman, D. (2010) A mathematical model of the sleep/wake cycle. J. Math. Biol. 60, 615-644.

Rhodes, F. \& Thompson, C. L. (1986) Rotation numbers for monotone functions on the circle. J. Lond. Math. Soc. 34, 360-368. 
Saper, C. B., Scammell, T. E. \& Lu, J. (2005) Hypothalamic regulation of sleep and circadian rhythms. Nature 437, 1257-1263.

Skeldon, A. C. \& Derks, G. (2017a) Nonsmooth Maps and the Fast-Slow Regulation of Sleep-Wake Regulation: Part I, Vol. 8, Birkhauser, Cham, pp. 167-170.

Skeldon, A. C., Derks, G. \& Booth, V. (2017b) Nonsmooth Maps and the Fast-Slow Regulation of Sleep-Wake Regulation: Part II, Vol. 8, Birkhauser, Cham, pp. 171-175.

Skeldon, A. C., Dijk, D.-J. \& Derks, G. (2014) Mathematical models for sleep-wake dynamics: Comparison of the two-process model and a mutual inhibition neuronal model. PLoS One 10, e103877.

Tamakawa, Y., Karashima, A., Koyama, Y., Katayama, N. \& NakaO, M. (2006) A quartet neural system model orchestrating sleep and wakefulness mechanisms. J. Neurophysiol. 95(4), 2055-2069.

Wever, R. (1979) The Circadian System of Man: Results of Experiments Under Temporal Isolation. Springer, New York. 